\title{
MoodPlayer: An Emotion Based Music Player
}

\author{
${ }^{1}$ Prof. Akshay Agrawal, ${ }^{2}$ Ms. Devanshi Shah, ${ }^{3}$ Ms. Pooja Shah, ${ }^{4}$ Mr. Nilang Trivedi \\ ${ }^{1}$ Asst.Professor, ${ }^{2,3,4}$ UG Student, ${ }^{1,2,3,4}$ Department of Information Technology, Universal College of \\ Engineering, Kaman, Maharashtra, India. \\ Iakshay.agarwal@universal.edu.in,2devanshishah522@gmail.com,3pooja.shah@universalcoe.edu.in, \\ 4nilang1998@gmail.com
}

Abstract - This report describes, songs, as expressional medium, has always been a consistent choice to portray and comprehend the feelings of a human being. A trustworthy system of emotion base classification can go a long way in helping us parse their significance. However, there are no optimum outcomes in the research field in emotion base classification systems. In this paper, we present an optimum cross-platform music player, MoodPlayer, which will suggest the songs according to the current mood of user. MoodPlayer provides smart and accurate mood base song recommendation by integrating the competence of emotion context reasoning within our versatile music suggestion system. Our music player works in two modules: Emotion Module, Song and Recommendation Module. A photo of user's face will be captured and taken as input in the initial steps of Emotion Module and using some algorithms the mood will be identified. The song and recommendation module proposes music to the end user by mapping their emotions to the mood type of the song and changes the playlist if the mood of the person is changed.

Keywords : Music Player, Emotion, Mood Based.

\section{INTRODUCTION}

Emotions of the humans are for understanding the mood and intentions, they can be understood from their expressions and speech [5]. There are different ways to manage the stress. Some may watch their favorite movie, some may meditate and some listen to their choice of music and songs [9]. According to the current research it is found that rhythms and songs induces emotional response into the listeners mind [1]. Personality traits and state of mind are directly correlated with musical preferences. Undoubtedly, a user's response to songs depends on a broad set of other factors, such as sex, age, culture, personal preferences, emotion and context (e.g. time of day or location). However, these other factors set aside, we are always able to classify songs as being happy, sad, enthusiastic or relaxed. Current research in emotion base recommender systems focuses on two main aspects, lyrics and audio features. Automated music segregation using some mood categories yields optimum results. Facial expressions are the most efficient and natural way of displaying emotions, feelings and mood. For the purpose of this paper, we categorize facial expressions into 4 different emotional categories, viz. joy, sorrow, anger and surprised. The main objective of this paper is to develop a cost-effective music player which automatically identifies the mood of current user and consequently generates a mood aware playlist and also check the emotion of the user while changing the songs. Least system resources are used while using this application. The emotion module determines the current mood of the user. The recommendation module integrates the outcome of the emotion module and suggests songs to the user. This system provides far better accuracy and performance than current systems. The person's state of mind is very necessary and identifying them through emotions that they display is also an important part [2].

\section{OBJECTIVE}

The main objective of this project is that people have the skills to view at someone's face and determine their current mood. This skill if studied by any computer, or a mobile can have significant utilization in the real world. As we all know, an emotion is the main aspect of communication, so the change of emotion can lead to many different aspects of communication [6]. Music, a tool for triggering feelings and emotions, is much stronger than language. Thus, listening to great songs can assist our mood to change from a negative to a positive. For instance, listening to energetic songs when an individual if feeling sad can assist him to come out of his unhappiness and start feeling good. With the increase in the number of songs and music an effective method of search and recommendation is getting more and more important. In music information retrieval, songs can be looked by title, genre, artist, etc. Keyword based search such as title or artist has a good outcome, but we have to know this data such as, artist, title, etc. in advance to search by keyword. In conventional music players, a person has to browse through his list and select songs according to their mood [3]. Real-time face detection and recognition systems 
have restricted capabilities due to the changing quality of images. Hence, researchers are still working for providing the solution. In the conventional music players, a user had to explicitly traverse the playlist he created and select songs that would comfort his current mood and emotions. This is a very tedious task and an individual often faced the problem to select the perfect songs.

\section{LITERATURE SURVEY}

\section{Automatic Facial Expression Recognition Using Features of Salient Facial Patches}

This paper proposes a completely unique framework for expression recognition by victimization look options of elite facial patches. a number of outstanding facial patches, reckoning on the position of facial landmarks, area unit extracted that area unit active throughout feeling stimulus. These active patches area unit more processed to get the salient patches that obtain discriminative options for classification of every try of expressions, thereby choosing completely different facial patches as salient for various try of expression categories. One-against-one classification methodology is adopted victimization these options.

\section{A survey of signal processing algorithms in brain computer interfaces based on electrical brain signals}

Brain-computer interfaces (BCIs) aim at providing a nonmuscular channel for causing commands to the external world exploitation the medical instrument activity or alternative electrophysiological measures of the brain perform. an important think about the successful operation of BCI systems is that the strategies accustomed method the brain signals. Within the BCI literature, however, there's no comprehensive review of the signal process techniques used. This work presents the first such comprehensive survey of all BCI styles exploitation electrical signal recordings printed before Jan 2006. Elaborated results from this survey area unit conferred and mentioned. The subsequent key analysis queries area unit addressed: what area unit the key signal process parts of a BCI, what signal process algorithms are utilized in BCIs and that signal process techniques have received a lot of attention.

\section{Facial Expression Recognition Using Eigen Spaces}

A lot of knowledge is sent by kinsmen within the type of countenance with the exception of simply what's spoken. Correct recognition of such expression has therefore become necessary for any trendy human pc interface. We have a tendency to gift here a technique of countenance recognition supported Eigen faces. It's a changed technique from the initial Eigen faces approach and starts out with the human vision as a typical indicator - by creating use of the quality JAFFE info and computes the expression contained by the image of a check face. It's a novel approach that directly classifies attest image as happiness to at least one of the six customary expressions - anger, disgust, fear, happy, unhappy or surprise with nice accuracy. During this paper, we have a tendency to gift with experimental proof the accuracy of such a method with analysis and discussion on additional ways that to enhance upon it.

4. An accurate and efficient statistical based approach for analyzing extracted facial expression features

The paper was majorly focused on the study of the changes in curvatures on the face and intensities of corresponding pixels of images. Artificial Neural Networks (ANN) was used in the classification extracted features into 6 major universal emotions like anger, disgust, fear, happy, sad, and surprise. A Scaled Conjugate Gradient back-propagation algorithm in correlation with two-layered feed forward neural network was used and was successful in obtaining a 92.2 recognition rate. In order to reduce the human effort and time needed for manual segregation of songs from a playlist, in correlation with different classes of emotions and moods, various approaches have been proposed.

\section{EXISTING SySTEM}

Varity of different methodologies have been suggested to categorize the behavioral and emotional state of the user. Music has also been segregated using lyrical summarization. Even though this method of classification is easier to implement but the result obtained from this method are not so accurate. Language barrier is another serious concern in the above mention method. Because it restricts the classification to a single language. [1]. We learn the utilization of $\mathrm{CNN}$ (convolutional neural networks) in the field of recognizing emotions. They are well known to imitate the brain and neural network when analyzing visuals; Thus, is executed to construct a computational model which categories emotion into 4 moods, namely, joy, sorrow, anger and surprised. The major drawback in this systems are that once the emotion is identified and the songs are played then the user has to manually change the song or go through the process again. These systems do not have the feature, that is if a user's mood changes then what to do? Their main idea is to keep the user's mood as it is by playing the songs [4].

\section{Problem Statement}

Using traditional music system, an end-user needs to manually traverse his playlist and select songs that might comfort his mood and emotions. Music listeners have difficult time creating to segregating the play-list explicitly when they have thousands of songs. It is also tough to keep track of all the songs: sometimes songs that are downloaded and never used, wasting a lot of device storage and forcing the user to search and delete songs manually. In today's world, with tremendous improvements in the area of technology and multimedia, various music players are being developed with functionalities like reverse, fast forward, playback, streaming, variable speed control with multicast streams and including volume adjustment, classification of songs by their genre etc. Although the mentioned features 
complete basic requirements of the user, the user still need to the tedious the task of explicitly traversing the playlist created before by him and select songs based on his current mood and emotions. The sequence of songs in a playlist is unique every time, and songs that a user wants to listen recursively might not be at the top of the list or it might get left out from the list. Currently, there are no such applications that let users to play music on-the-go without selecting songs explicitly or from a created playlist and play songs according to the change in their mood.

\section{Proposed SysteM}

The very first and initial phase is detecting the Facial Expression of the user through Face Detection. The process of extracting the various components like ears, eyes, mouth from a face is known as facial feature extraction. It is very crucial to start the process of expression recognition. Camera module extracts the facial features and detects the mood of the user depending on the expression. The Recommendation module lets you integrate functions which are carried out on the YouTube website into your website or application. This feature helps us to store songs in the form of playlist. This playlist can be categorized in various forms depending on the need. Using this feature, we can play songs based on the mood categorized.

\section{IMPLEMENTATION}

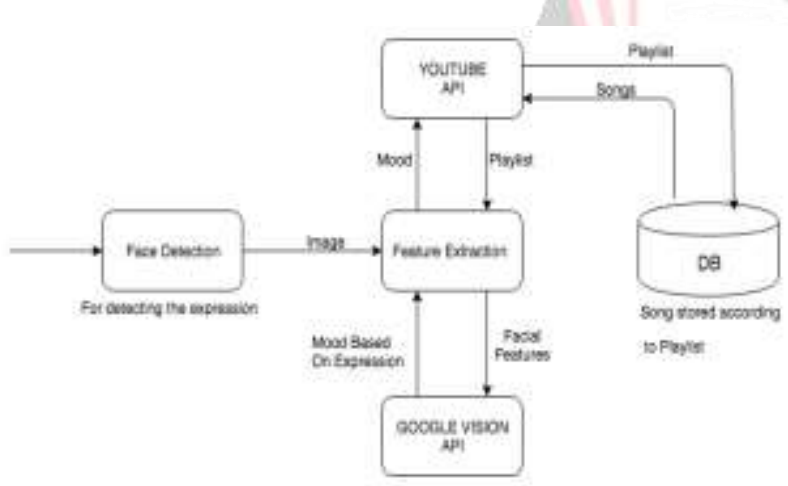

The very first and initial phase is detecting the Facial Expression of the user through Face Detection. The process of extracting the various components like ears, eyes, mouth from a face is known as facial feature extraction. It is very crucial to start the process of expression recognition or face recognition. Camera module allows developers to integrate detection features within applications, including image labeling, face detection. Camera Module extracts the facial features and detects the mood of the user depending on the expression. This Recommender module lets you integrate functions which are carried out on the YouTube website into your website or application. Using this feature, we can play songs based on the mood categorized. Once the person listens to the songs and after a while the mood changes then the habit of skipping songs can help in understanding the change in mood of the person which leads to the recommender module to change the playlist.

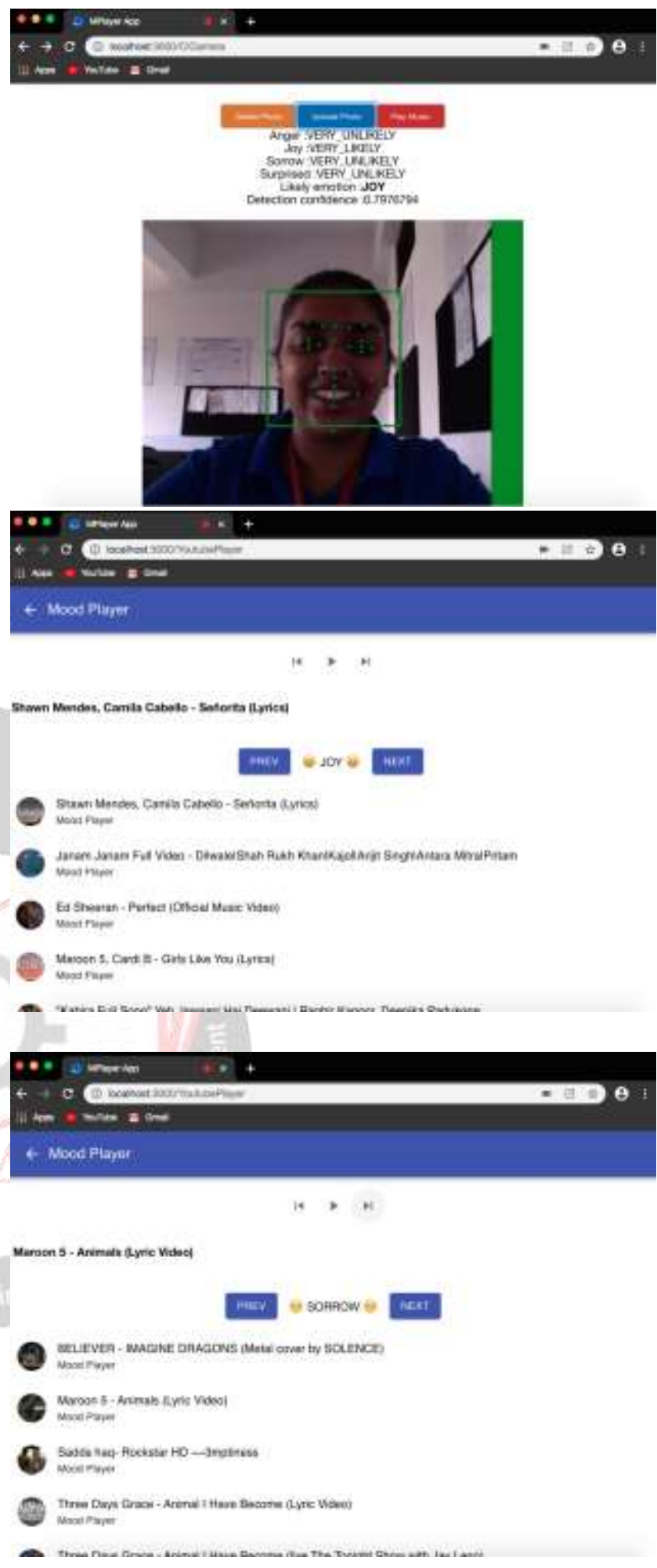

VIII. Advantages

The following are the advantages of our system

1. As the MoodPlayer is a Progressive Web Application, it does not require any installation on the laptop, desktop or on a mobile phone.

2. The user's efforts to traverse through the playlist and selecting a song is omitted as the songs will play according to the user's current mood.

3. One of the major advantages over existing system is once the mood of user is recognized it may 
happen that the mood of user might change while listening to the songs. The existing system are not capable of changing their playlist after mood has been recognized. Whereas, MoodPlayer is capable of changing the playlist if user skips some song of same genre.

4. The user interface of the MoodPlayer is very user friendly and easy to understand.

\section{Conclusion}

The main objective of this paper was to implement an emotion base music recommendation system using the functionalities of facial expression recognition. Other than theoretical background, above work provides different ways to deal with the problems and operates emotion base music player. Above mentioned system is able to operate on input pictures of the user's face and determine the mood and then play music considering the emotion taken as a input from pervious module and also recommend songs that soothes the mood of the user. In the future work, we would like to work on giving more number of moods such as Disgust, Fear, Neutral as the output and not only four, also, the rate and accuracy of expression detection to improve [8]. We would like to develop a system which automatically segregates the songs and music according to its genre. These new modules integrated with current developed system will improve the user satisfaction.

\section{REFERENCES}

[1] S. Gilda, H. Zafar, C. Soni and K. Waghurdekar, "Smart music player integrating facial emotion recognition and music mood recommendation," 2017 International Conference on Wireless Communications, Signal Processing and Networking (WiSPNET), Chennai, 2017, pp. 154-158.

[2] R. Ramanathan, R. Kumaran, R. Ram Rohan, R. Gupta and V. Prabhu, "An Intelligent Music Player Based on Emotion Recognition," 2017 2nd International Conference on Computational Systems and Information Technology for Sustainable Solution (CSITSS), Bangalore, 2017, pp. 1-5.

[3] Iyer, Aurobind \& Pasad, Viral \& Sankhe, Smita \& Prajapati, Karan. (2017). Emotion based mood enhancing music recommendation. 1573-1577. 10.1109/RTEICT.2017.8256863.

[4] Shubham S. Jagtap, Prof. Pramod T. Talole, Jayshri V. Kharat, Pooja R. Bali, "INTELLIGENT MUSIC PLAYER USING EMOTION RECOGNITION” 2018 INTERNATIONAL JOURNAL OF CURRENT ENGINEERING AND SCIENTIFIC RESEARCH (IJCESR) 2018 pp. 2393-8374.

[5] Vishal A. Desai, Amith A. Kadam, Amol Baviskar, "Emotion Analysis Based Intelligent Music Player using Android System" 2016 International Journal on Recent and Innovation Trends in Computing and Communication 2016 pp. 917 - 920.

[6] M. Xiaoxi, L. Weisi, H. Dongyan, D. Minghui and H. Li, "Facial emotion recognition," 2017 IEEE 2nd International Conference on Signal and Image Processing (ICSIP), Singapore, 2017, pp. 77-81.

[7] A. A. Varghese, J. P. Cherian and J. J. Kizhakkethottam, "Overview on emotion recognition system," 2015 International Conference on SoftComputing and Networks Security (ICSNS), Coimbatore, 2015, pp. 1-5.

[8] H. Lee and K. Hong, "A study on emotion recognition method and its application using face image," 2017 International Conference on Information and Communication Technology Convergence (ICTC), Jeju, 2017, pp. 370-372.

[9] K. Chankuptarat, R. Sriwatanaworachai and S. Chotipant, "Emotion-Based Music Player," 2019 5th International Conference on Engineering, Applied Sciences and Technology (ICEAST), Luang Prabang, Laos, 2019, pp. 1-4.

$x^{2}$

